\title{
A new metric for rotating charged Gauss-Bonnet black holes in AdS spaces
}

\author{
De-Cheng Zou ${ }^{1 *}$, Zhan-Ying Yang ${ }^{1 \dagger}$, Rui-Hong Yue ${ }^{2 \ddagger}$ and Tian-Yi Yu ${ }^{2}$ \\ ${ }^{1}$ Department of Physics, Northwest University, Xi'an, 710069, China \\ ${ }^{2}$ Faculty of Science, Ningbo University, Ningbo, 315211, China
}

(Dated: November 8, 2018)

\begin{abstract}
This paper presents a new metric for slowly rotating charged Gauss-Bonnet black holes in higher dimensional anti-de Sitter spaces. Taking the angular momentum parameter $a$ up to second order, the slowly rotating charged black hole solutions are obtained by working directly in the action.
\end{abstract}

\section{INTRODUCTION}

The higher derivative gravity theories have gained much interest during the past years. It is believed that Einstein's action is only an effective gravitational action valid for small curvatures or low energies [1]. Considering the fundamental nature of quantum gravity, one sees that the action will be modified by higher derivative interactions in the the renormalization of quantum field theory in curved spacetimes [2-4]. Besides, these higher derivative terms can be seen in the construction of the low-energy effective action of string [5] 8$]$. The so-called Lovelock gravity is quite special [9]. Except for the advantage that the equations of motion of the Lovelock gravity, as the case of the Einstein's general relativity, do not contain terms with more than second derivatives of metric, the Lovelock gravity has been shown to be free of ghost when expanding on a flat space, evading any problems with unitarity [10, 11]. The Gauss-Bonnet terms appear as the first higher derivative curvature correction term to general relativity. The black hole solutions in Gauss-Bonnet gravity were first discovered by Boulware and Deser [12] and Wheeler [13], independently. The thermodynamics of the uncharged static spherically Gauss-Bonnet black hole solutions have been considered in Refs

\footnotetext{
* Email:zoudecheng789@163.com

$\dagger$ Email:zyyang@nwu.edu.cn

‡Email:yueruihong@nbu.edu.cn
} 
[14 16] and of charged solutions in Ref [17]. Recently the quasinormal mode of a scalar field in five dimensional Lovelock black hole spacetime for different angular quantum numbers $l$ has been obtained in [18]. Liu [19] studied the electromagnetic perturbations of black holes in Gauss-Bonnet gravity.

It is of interest to generalize these static Gauss-Bonnet solutions by including the effects of rotation. This problem has been considered recently in [23] within a perturbative approach. It is worth mentioning that, for the Gauss-Bonnet action, the resulting field equations, obtained after variation with respect to the metric tensor, have seven terms. While the resulting field equation for third order Lovelock gravity contains thirty-four terms. However, by working directly in the action, the exact static Gauss-Bonnet black hole solutions were obtained [12 16], and third order Lovelock black hole solutions in [20 22]. Using the same approach, in this paper, we want to obtain the slowly rotating black hole solutions in Lovelock gravity. Apparently the lowest level contribution of rotation should be proportional to $a^{2}$. Hence, linearly dependent on $a$, the metric demonstrated in Ref [23] can not be adopted in this case. So, we need to find a proper metric ansatz up to $a^{2}$.

This paper is organized as follows. In Section 2, we present a new form metric and obtain slowly rotating charged black hole solutions by working directly in the action. Then we discuss some related thermodynamic properties of black holes. Section 3 is devoted to a summary of the results.

\section{SLOWLY ROTATING CHARGED GAUSS-BONNET BLACK HOLES}

\section{A. A new metric and Rotating black holes}

In general relativity, the higher dimensional generalization of the Kerr-AdS black hole solutions can be found in [24, 25]. It is expressed as in the Boyer-Lindpuist type coordinate

$$
\begin{aligned}
d s^{2}= & -\frac{\Delta_{r}}{\Sigma}\left(d t-\frac{a \sin ^{2} \theta}{\Xi} d \phi\right)^{2}+\frac{\Sigma}{\Delta_{r}} d r^{2}+\frac{\Sigma}{\Delta_{\theta}} d \theta^{2}+\frac{\Delta_{\theta} \sin ^{2} \theta}{\Sigma}\left(a d t-\frac{r^{2}+a^{2}}{\Xi} d \phi\right)^{2} \\
& +r^{2} \cos ^{2} \theta d \Omega_{D-4}^{2},
\end{aligned}
$$


where

$$
\begin{aligned}
& \Sigma=r^{2}+a^{2} \cos ^{2} \theta, \quad \Delta_{r}=\left(r^{2}+a^{2}\right)\left(1+\frac{r^{2}}{l^{2}}\right)-m r^{5-D}, \\
& \Delta_{\theta}=1-\frac{a^{2}}{l^{2}} \cos ^{2} \theta, \quad \Xi=1-\frac{a^{2}}{l^{2}} .
\end{aligned}
$$

The parameters $m$ and $a$ are related to the mass and angular momentum of the black hole, as we shall see.

If we consider the metric Eq. (11) up to the second order in the parameter $a$, the components of the metric are expressed as follows:

$$
\begin{aligned}
g_{t t} & =-\left[1-\frac{m}{r^{D-3}}+\frac{r^{2}}{l^{2}}+\frac{a^{2}}{l^{2}} \sin ^{2} \theta+\frac{a^{2} m \cos ^{2} \theta}{r^{D-1}}\right]+\mathcal{O}\left(a^{4}\right), \\
g_{r r} & =\frac{1+a^{2} \cos ^{2} \theta / r^{2}}{1-\frac{m}{r^{D-3}}+\frac{r^{2}}{l^{2}}}-\frac{a^{2}}{\left(1-\frac{m}{r^{D-3}}+\frac{r^{2}}{l^{2}}\right)^{2}}\left(\frac{1}{l^{2}}+\frac{1}{r^{2}}\right)+\mathcal{O}\left(a^{4}\right), \\
g_{\theta \theta} & =\left(r^{2}+a^{2} \cos ^{2} \theta+\frac{a^{2} r^{2}}{l^{2}} \cos ^{2} \theta\right)+\mathcal{O}\left(a^{4}\right), \\
g_{\phi \phi} & =\left[r^{2}+a^{2}+a^{2} \sin ^{2} \theta\left(\frac{m}{r^{D-3}}-\frac{r^{2}}{l^{2}}\right)+\frac{2 a^{2} r^{2} \sin ^{2} \theta}{l^{2}}\right] \sin ^{2} \theta+\mathcal{O}\left(a^{4}\right), \\
g_{t \phi} & =2 a \sin ^{2} \theta\left(\frac{r^{2}}{l^{2}}-\frac{m}{r^{D-3}}\right)+\mathcal{O}\left(a^{3}\right) .
\end{aligned}
$$

Setting $a=0$, we find that metric Eq. (2) reduce to the static case. It is known that the static black hole solution in D dimensional spacetimes in general relativity is $f(r)=1-\frac{m}{r^{D-3}}+\frac{r^{2}}{l^{2}}$. Replacing $1-\frac{m}{r^{D-3}}+\frac{r^{2}}{l^{2}}$ with $f(r)$, we obtain a new metric when all terms involving $a^{3}$ and higher powers in $a$ are ignored

$$
\begin{aligned}
d s^{2}= & -\left[f(r)+\frac{a^{2}}{l^{2}}+\frac{a^{2}(1-f(r)) \cos ^{2} \theta}{r^{2}}\right] d t^{2} \\
& +\left[\frac{1}{f(r)}+\frac{a^{2}\left(\cos ^{2} \theta f(r)-1\right)}{r^{2} f(r)^{2}}-\frac{a^{2}}{l^{2} f(r)^{2}}\right] d r^{2} \\
& +\left(r^{2}+a^{2} \cos ^{2} \theta+\frac{a^{2} r^{2}}{l^{2}} \cos ^{2} \theta\right) d \theta^{2}+\left[r^{2}+a^{2}\right. \\
& \left.+a^{2}(1-f(r)) \sin ^{2} \theta+\frac{2 a^{2} r^{2} \sin ^{2} \theta}{l^{2}}\right] \sin ^{2} \theta d \phi^{2} \\
& +2 a r^{2} p(r) \sin ^{2} \theta d t d \phi+r^{2} \cos ^{2} \theta d \Omega_{D-4}^{2},
\end{aligned}
$$

where parameter $a^{2}$ is a small quantity and the functions $f(r)$ and $p(r)$ satisfy the relation $p(r)=\frac{-1+f(r)}{r^{2}}$. Simplification for future, we order $f(r)$ and $p(r)$ are two independent variables. Besides, taking the parameter $a$ up to the first order, the metric Eq. (3) becomes the counterpart in Ref [23]. 
The action for Gauss-Bonnet theory with negative cosmological constant $\Lambda=-(D-$ 1) $(D-2) / l^{2}$ in $D$ dimensions is given by [23]

$$
\mathcal{I}=\frac{1}{16 \pi G} \int d^{D} x \sqrt{-g}\left(-2 \Lambda+R+\alpha \mathcal{L}_{G B}-4 \pi G F_{\mu \nu} F^{\mu \nu}\right),
$$

where $\alpha$ is the Gauss-Bonnet coefficient with dimension (length) ${ }^{2}$ and is rescaled with $\frac{\tilde{\alpha}}{(D-3)(D-4)}$ in future. Note that the Lagrangian of Lovelock gravity is the sum of dimensionally extended Euler densities, and then, the Lagrangian of Gauss-Bonnet term also can be expressed as [15]

$$
\mathcal{L}_{G B}=\frac{1}{4} \delta_{c_{1} b_{1} c_{2} d_{2}}^{a_{1} b_{1} a_{2} b_{2}} R_{a_{1} b_{1}}^{c_{1} d_{1}} R_{a_{2} b_{2}}^{c_{2} d_{2}}
$$

Here, all non-vanishing components of Riemann tensors (up to $a^{2}$ ) can be expressed as

$$
R_{k l}^{i j}=\bar{R}_{k l}^{i j}+\tilde{R}_{k l}^{i j}
$$

where $\bar{R}_{k l}^{i j}$ is in the absence of $a$ and $\tilde{R}_{k l}^{i j}$ is proportional to $a^{2}$. Moreover, based on the relationship of indexes, these Riemann tensors also can be classified into three groups: (I) diagonal form $R_{\hat{i} \hat{j} \hat{j}}^{\hat{i}}$, (II) off-diagonal form proportional to $a$ and (III) off-diagonal form proportional to $a^{2}$. For group (I), we find that the Riemann tensors $\bar{R}_{12}^{12}, \bar{R}^{1 i}{ }_{1 i}, \bar{R}^{2 i}{ }_{2 i}$ and $\bar{R}_{i j}^{i j}$ are all absent from parameter $a$. They can be represented as $\bar{R}_{12}^{12}=-\frac{f^{\prime \prime}(r)}{2}$, $\bar{R}^{1 i}{ }_{1 i}=-\frac{f^{\prime}(r)}{2 r}, \bar{R}^{2 i}{ }_{2 i}=-\frac{f(r)}{2 r}$ and $\bar{R}^{i j}{ }_{i j}=\frac{1-f(r)}{r^{2}}(3 \leq i<j \leq D)$ which is independent to the dimensions $D$. Some of key steps are given in appendix. Denoted the Lagrangian of Gauss-Bonnet term from the contribution of the case (I) by $\mathcal{L}_{d}$, it can be written as

$$
\begin{aligned}
\mathcal{L}_{d} & =\overline{\mathcal{L}}_{d}+\tilde{\mathcal{L}}_{d} \\
& \left.=\frac{1}{4} \delta_{c_{1} b_{1} c_{2} d_{2}}^{a_{1} b_{1} a_{2} b_{2}} \bar{R}_{a_{1} b_{1}}^{c_{1} d_{1}} \bar{R}_{a_{2} b_{2}}^{c_{2} d_{2}}+2 \bar{R}_{a_{1} b_{1}}^{c_{1} d_{1}} \tilde{R}_{a_{2} b_{2}}^{c_{2} d_{2}}\right) \\
& =\bar{A}_{0}+\bar{A}_{1}+\bar{A}_{2}+\bar{A}_{3}+\bar{A}_{4}+\tilde{B}_{1}+\tilde{B}_{2}+\tilde{B}_{3}+\tilde{B}_{4}
\end{aligned}
$$

where

$$
\begin{aligned}
\bar{A}_{0} & =\frac{1}{4} \delta_{c_{1} b_{1} c_{2} d_{2}}^{a_{1} b_{1} a_{2} b_{2}} \bar{R}_{a_{1} b_{1}}^{c_{1} d_{1}} \bar{R}_{a_{2} b_{2}}^{c_{2} d_{2}} \\
& =(D-2)(D-3)(D-4)(D-5)\left(\bar{R}_{i j}^{i j}\right)^{2}, \quad(i, j \geq 3) \\
\bar{A}_{1} & =2 \delta_{c_{1} b_{1} 12}^{a_{1} b_{1} 12} \bar{R}_{a_{1} b_{1}}^{c_{1} d_{1}} \bar{R}_{12}^{12}=4(D-2)(D-3) \bar{R}_{12}^{12} \bar{R}^{i j}{ }_{i j}, \\
\bar{A}_{2} & =\frac{1}{4} \delta_{1 i 2 j}^{1 i 2 j} \bar{R}_{1 i}^{1 i} \bar{R}_{2 j}^{2 j}=8(D-2)(D-3) \bar{R}_{1 i}^{1 i} \bar{R}_{2 j}^{2 j}, \\
\bar{A}_{3} & =\frac{1}{4} \delta_{1 i j k}^{1 i j k} \bar{R}_{j k}^{j k} \bar{R}_{1 i}^{1 i}=4(D-2)(D-3)(D-4) \bar{R}_{1 i}^{1 i} \bar{R}_{j k}^{j k}, \quad \\
\bar{A}_{4} & =\frac{1}{4} \delta_{2 i j k}^{2 i j k} \bar{R}_{j k}^{j k} \bar{R}_{2 i}^{2 i}=4(D-2)(D-3)(D-4) \bar{R}_{2 i}^{2 i} \bar{R}_{j k}^{j k}, \quad(i, j, k \geq 3)
\end{aligned}
$$


and

$$
\begin{aligned}
\tilde{B}_{1} & =4(D-2)(D-3) \tilde{R}_{12}^{12} \times \bar{R}_{34}^{34}, \\
\tilde{B}_{2} & =4 \sum_{i=3}^{D} \tilde{R}^{1 i}{ }_{1 i} \times\left[2(D-3) \bar{R}^{24}{ }_{24}+(D-3)(D-4) \bar{R}^{45}{ }_{45}\right], \\
\tilde{B}_{3} & =4 \sum_{i=3}^{D} \tilde{R}_{2 i}^{2 i} \times\left[2(D-3) \bar{R}_{14}^{14}+(D-3)(D-4) \bar{R}_{45}^{45}\right], \\
\tilde{B}_{4} & =8 \sum_{i>j=3}^{D} \tilde{R}^{i j}{ }_{i j} \times\left[\bar{R}_{12}^{12}+(D-4)\left(\bar{R}_{13}^{13}+\bar{R}_{25}^{25}\right)\right. \\
& \left.+\frac{(D-4)(D-5)}{2} \bar{R}_{k l}^{k l}\right], \quad(k>l \geq 4) .
\end{aligned}
$$

Inspecting the off-diagonal Riemann tensors, we calculate the parts of Lagrangian from the off-diagonal Riemann tensors in case (II)

$$
\mathcal{L}_{o d}=\mathcal{L}_{o d 1}+\mathcal{L}_{o d 2}+\mathcal{L}_{o d 3}
$$

where

$$
\begin{aligned}
& \mathcal{L}_{\text {od } 1}=4\left(R^{12}{ }_{34} R^{34}{ }_{12}+R^{13}{ }_{24} R^{24}{ }_{13}+R^{14}{ }_{23} R^{23}{ }_{14}\right), \\
& \mathcal{L}_{\text {od } 2}=\sum_{i=3}^{D} 4\left(R^{12}{ }_{24} R^{i 4}{ }_{1 i}+R^{24}{ }_{12} R^{1 i}{ }_{i 4}\right), \\
& \mathcal{L}_{\text {od } 3}=\sum_{i, j=3}^{D} 4\left(R^{1 i}{ }_{i 4} R^{j 4}{ }_{1 j}+R^{1 j}{ }_{j 4} R^{i 4}{ }_{1 i}\right), \quad(i>j \geq 3) .
\end{aligned}
$$

It is interesting to note that the contribution of off-diagonal Riemann tensors in case (III) vanishes following the properties of the Kronecker delta symbol. In addition, the Ricci scalar $R$ is given by

$$
R=\bar{R}+\tilde{R},
$$

where $\bar{R}$ is equal to $-\frac{6 f^{\prime}(r)}{r}+\frac{6(1-f(r))}{r^{2}}-f^{\prime \prime}(r)$ and $\tilde{R}$ is proportional to $a^{2}$ and shown in appendix.

Since the black hole rotates along the direction $\phi$, it will generate a magnetic field. If we considering this effect, the gauge potential can be chosen

$$
A_{\mu} d x^{\mu}=A_{t} d t+A_{\phi} d \phi
$$


where $A_{t}=Q h(r)+a^{2} \cos ^{2} \theta Q k(r), \quad A_{\phi}=-a \sin ^{2} \theta Q c(r)$. Therefore the nonzore contravariant component of the electromagnetic field tensor can be written as

$$
\begin{aligned}
& F_{t r}=-Q h^{\prime}(r)-a^{2} \cos ^{2} \theta Q k^{\prime}(r), \quad F_{t \theta}=a^{2} \sin (2 \theta) Q k(r) \\
& F_{r \phi}=-a Q c^{\prime}(r) \sin ^{2} \theta, \quad F_{\theta \phi}=-a Q c(r) \sin (2 \theta) .
\end{aligned}
$$

By substituting the ansatz Eq. (3) and tensors of electromagnetic field Eq. (13) into the action Eq. (44), the action becomes

$$
\mathcal{I}=\mathcal{I}(f(r), p(r), h(r), k(r), c(r)) \text {. }
$$

Firstly, varying the action Eq. (14) with regard to the function $k(r)$, we find the solution

$$
h^{\prime \prime}(r) r+(D-2) h^{\prime}(r)=0 .
$$

Hence, we obtain

$$
h(r)=\frac{C_{2}}{r^{D-3}}+C_{1}
$$

where $C_{1}$ and $C_{2}$ are two integration constants. We choose $C_{1}=0$ and $C_{2}=\frac{1}{4(D-3) \pi}$ and then the function $h(r)$ can be written as $h(r)=\frac{1}{4 \pi(D-3) r^{D-3}}$. Then, the variation of the action Eq. (14) with regard to $c(r)$ becomes

$$
\left[r^{D-4} f(r) c^{\prime}(r)\right]^{\prime}-2(D-3) r^{D-6} c(r)+\frac{p^{\prime}(r)}{4 \pi}=0
$$

Taking the variation of the action Eq. (14) with regard to the function $p(r)$, we arrive at

$$
\begin{aligned}
0 & =\left[2 \tilde{\alpha} f(r)-2 \tilde{\alpha}-r^{2}\right] r^{2} f(r) p^{\prime \prime}(r) \\
& +\left[2(D-2) \tilde{\alpha}(f(r)-1)-D r^{2}+2 \tilde{\alpha} r f^{\prime}(r)\right] r f(r) p^{\prime}(r) \\
& +\left\{( D - 2 ) \left[-f^{\prime}(r) r^{3}+(1-f(r))\left((D-3) r^{2}-2 \tilde{\alpha} r f^{\prime}(r)\right)+(D-5) \tilde{\alpha}(1-f(r))^{2}\right.\right. \\
& \left.\left.+\frac{(D-1) r^{4}}{l^{2}}\right]-8 \pi G Q^{2} r^{4} h^{\prime}(r)\right\} p(r)+16 \pi G Q^{2} r h^{\prime}(r) c^{\prime}(r) .
\end{aligned}
$$

If supposing the coefficient of function $p(r)$ vanishes, we find that, for $h(r)=\frac{1}{4 \pi(D-3) r^{D-3}}$, the function $f(r)$ is obtained

$$
f(r)=1+\frac{r^{2}}{2 \tilde{\alpha}}\left(1-\sqrt{1-\frac{4 \tilde{\alpha}}{l^{2}}+\frac{4 \tilde{\alpha} m}{r^{D-1}}-\frac{2 \tilde{\alpha}}{\pi(D-2)(D-3)} \frac{G Q^{2}}{r^{2 D-4}}}\right) .
$$


Moreover, the Eq.(18) reduces to

$$
r^{D}[1+2 \tilde{\alpha} \omega(r)] p^{\prime}(r)+4 G Q^{2} c(r)+C_{3}=0,
$$

where $\omega(r)=(1-f(r)) / r^{2}$. Based on the Ref. [23], the function $p(r)$ is given by

$$
p(r)=\frac{1}{2 \tilde{\alpha}}\left(1-\sqrt{1-\frac{4 \tilde{\alpha}}{l^{2}}+\frac{4 \tilde{\alpha} m}{r^{D-1}}-\frac{2 \tilde{\alpha}}{\pi(D-2)(D-3)} \frac{G Q^{2}}{r^{2 D-4}}}\right) .
$$

and $c(r)$ is

$$
c(r)=-\frac{1}{4(D-3) \pi r^{D-3}},
$$

by taking constant $C_{3}=(D-1) m$. Apparently the functions $f(r)$ and $p(r)$ satisfy the relationship $p(r)=-(1-f(r)) / r^{2}$.

Furthermore, varying the action with regard to the electromagnetic field $h(r)$, we have

$$
(D-1)+2(D-2)(D-3) \pi r^{D} k^{\prime}(r)+2(D-3) \pi r^{D+1} k^{\prime \prime}(r)=0 .
$$

We can obtain $k(r)=-\frac{1}{4 \pi(D-3) r^{D-1}}$ where integral constants are all ignored. Therefore, the tensor of electromagnetic field are given as

$$
\begin{aligned}
F_{t r} & =\frac{Q}{4 \pi r^{D-2}}-\frac{(D-1)}{4 \pi(D-3) r^{D}} a^{2} Q \cos ^{2} \theta, \quad F_{t \theta}=-\frac{a^{2} Q}{4 \pi(D-3) r^{D-1}} \sin (2 \theta) \\
F_{r \phi} & =-\frac{a Q}{4 \pi r^{D-2}} \sin ^{2} \theta, \quad F_{\theta \phi}=\frac{a Q}{4 \pi(D-3) r^{D-3}} \sin (2 \theta) .
\end{aligned}
$$

Furthermore, it is easy to verify that the expressions for functions $f(r)$ Eq. (20), $p(r)$ Eq. (21), $c(r)$ Eq. (22) and $h(r)=-k(r) r^{2}=\frac{1}{4 \pi(D-3) r^{D-3}}$ still satisfy the variation of action Eq. (4) respecting to $f(r)$.

\section{B. Thermodynamics of black holes}

The Killing vectors can be used to give a physical interpretation of the parameter $m$ and a. Following the analysis given in [25 28], one can obtain coordinate-independent definitions for these parameters. We have the integral

$$
M=-\frac{1}{16 \pi G} \frac{D-2}{D-3} \oint \xi_{(t)}^{\mu ; \nu} d^{D-2} \Sigma_{\mu \nu}, \quad J=\frac{1}{16 \pi G} \oint \xi_{(\phi)}^{\mu ; \nu} d^{D-2} \Sigma_{\mu \nu},
$$


where the integrals are taken over the $(D-2)$-sphere at spatial infinity,

$$
d^{D-2} \Sigma_{\mu \nu}=\frac{1}{(D-2) !} \sqrt{-g} \epsilon_{\mu \nu \alpha \beta \gamma} d x^{i_{1}} \wedge d x^{i_{2}} \wedge \ldots d x^{i_{D-2}}
$$

To justify the definitions Eq. (25), we can calculate the integrands in the asymptotic region $r \rightarrow \infty$. For the dominant terms in the asymptotic expansion we have

$$
\xi_{(t)}^{t ; r}=\frac{m(D-3)}{2 r^{D-2}}+\mathcal{O}\left(\frac{1}{r^{D}}\right), \quad \xi_{(\phi)}^{t ; r}=-\frac{a m(D-1) \sin ^{2} \theta}{2 r^{D-2}}+\mathcal{O}\left(\frac{1}{r^{D}}\right) .
$$

We perform the integration over a $(D-2)$-sphere at $r \rightarrow \infty$. It gives

$$
M=\frac{m(D-2) \Sigma_{k}}{16 \pi G}, \quad J=\frac{2 M a}{D-2} .
$$

In addition, if $a=0$, the black hole does not rotate and the metric represents a spherically symmetric charged black hole with a spherically symmetric electric field. If the black hole rotates $a \neq 0$, the electric field is supplemented by a magnetic field due to the dragging of the inertial reference frames into rotational motion around the black hole.

\section{CONCLUDING REMARKS}

In present paper, we proposed an new metric and then obtained the slowly rotating charged Gauss-Bonnet black hole solutions in anti-de Sitter spaces by working directly in the action. Besides the function $f(r)$, the diagonal components of the metric also involve $a^{2}$. Moreover, the $g_{t \phi}$ is proportional to $r^{2} p(r)$ as to make the equation for $p(r)$ much simple. Since the black hole rotates along the direction $\phi$, vector potential has an extra nonradial component $A_{\phi}=-a \sin ^{2} \theta Q c(r)$ and the $t$ component is written as $A_{t}=Q h(r)+$ $a^{2} \cos ^{2} \theta Q k(r)$. By taking variation of the action respecting to the functions $p(r), f(r), c(r)$, $h(r)$ and $k(r)$, respectively, we got the exact form for function $p(r)$, while the function $f(r)$ still kept the form of the static solutions. Moreover, the exact expressions for $c(r), h(r)$ and $k(r)$ have been expressed as $c(r)=-h(r)=k(r) r^{2}=-\frac{1}{4 \pi(D-3) r^{D-3}}$.

\section{APPENDIX}

From the metric Eq. (3), we obtain some of the intermediate steps of the calculation. 
Riemann tensors. The non-vanishing Riemann tensors can be classified into three groups: (I) diagonal form $R_{\hat{i} \hat{j} \hat{j}}^{\hat{i}}$, (II) off-diagonal form proportional to $a$ and (III) off-diagonal form proportional to $a^{2}$.

group (I):

$$
\begin{aligned}
R_{12}^{12} & =\bar{R}_{12}^{12}+\tilde{R}_{12}^{12}, \quad \bar{R}_{12}^{12}=-\frac{f(r)^{\prime \prime}}{2} \\
\tilde{R}_{12}^{12} & =a^{2}\left\{\left[\left(-1+\frac{r f(r)^{\prime}+r^{2} f(r)^{\prime \prime}}{2 f(r)}-\frac{r^{2} f(r)^{\prime 2}}{4 f(r)^{2}}\right) p(r)^{2}\right.\right. \\
& +\left(\frac{r^{2} f(r)^{\prime} p(r)^{\prime}}{f(r)}-5 r p(r)^{\prime}-r^{2} p(r)^{\prime \prime}\right) \frac{p(r)}{2}-\frac{r^{2} p(r)^{\prime 2}}{4}+\frac{f(r)^{\prime}-r f(r)^{\prime \prime}}{2 r^{3} f(r)} \\
& \left.\left.+\frac{f(r)^{\prime 2}}{4 r^{2} f(r)^{2}}\right] \sin ^{2} \theta+\left[\frac{-2 f(r)^{\prime}}{r^{3}}-\frac{3(1-f(r))}{r^{4}}+\frac{f(r)^{\prime \prime}}{2 r^{2}}\right] \cos ^{2} \theta\right\},
\end{aligned}
$$

$$
\begin{aligned}
R_{1 i}^{1 i} & =\bar{R}_{1 i}^{1 i}+\tilde{R}_{1 i}^{1 i}, \quad \bar{R}_{1 i}^{1 i}=-\frac{f(r)^{\prime}}{2 r}, \quad(3 \leq i \leq D), \\
\tilde{R}_{13}^{13} & =\left\{-\frac{p(r)^{2}}{f(r)}+\frac{1}{f(r) r^{4}}+\frac{f(r)^{\prime}}{r^{3}}-\frac{f(r)}{r^{4}}+\left[-\frac{r p(r)^{2} f(r)^{\prime}}{2 f(r)}+\frac{r p(r) p(r)^{\prime}}{2}\right.\right. \\
& \left.\left.-\frac{1+f(r)}{r^{4}}-\frac{f(r)^{\prime}}{2 f(r) r^{3}}+\frac{2}{r^{4} f(r)}-\frac{2 p(r)^{2}}{f(r)}+\frac{f(r)^{\prime}}{r^{3}}+p(r)^{2}\right] \sin ^{2} \theta\right\} a^{2} \\
\tilde{R}_{14}^{14} & =\left\{\left[-p(r)^{2}-r p(r)^{\prime} p(r)-\frac{r^{2} p(r)^{\prime 2}}{4}+\frac{f(r)^{\prime 2}}{r^{4}}-\frac{f(r)^{\prime}}{2 r^{3} f(r)}+\frac{f(r)^{\prime}}{4 r^{2}}\right.\right. \\
& \left.\left.-\frac{f(r)^{\prime} f(r)}{2 r^{3}}+\frac{r f(r)^{\prime} p(r)^{2}}{2 f(r)}\right] \sin ^{2} \theta+\frac{f(r)^{\prime}}{r^{3}}+\frac{-p(r)^{2} r^{4}+1-f(r)^{2}}{f(r) r^{4}} \cos ^{2} \theta\right\} a^{2} \\
\tilde{R}_{1 j}^{1 j}= & \left\{\left[\frac{p(r)^{2}}{f(r)}-p(r)^{2}-\frac{f(r)^{\prime}}{2 r^{3} f(r)^{\prime}}-\frac{1}{r^{4} f(r)}+\frac{f(r)^{\prime} p(r)^{2}}{2 f(r)}-\frac{r p(r)^{\prime} p(r)}{2}\right] \sin ^{2} \theta\right. \\
+ & {\left.\left[\frac{f(r)^{\prime}}{2 r^{3}}-\frac{f(r)}{r^{4}}\right] \cos ^{2} \theta+\frac{1}{r^{4}}\right\} a^{2}, \quad(5 \leq j \leq D), }
\end{aligned}
$$

$$
\begin{aligned}
R_{2 i}^{2 i} & =\bar{R}_{2 i}^{2 i}+\tilde{R}_{2 i}^{2 i}, \quad \bar{R}_{2 i}^{2 i}=-\frac{f(r)}{2 r}, \quad(3 \leq i \leq D), \\
\tilde{R}_{23}^{23} & =\tilde{R}_{2 j}^{2 j}=a^{2}\left[\frac{f(r)^{\prime}}{r^{3}}+\frac{2(1-f(r))}{r^{4}}\right] \cos ^{2} \theta, \quad(5 \leq j \leq D), \\
\tilde{R}_{24}^{24} & =\left\{\left[\frac{f(r)^{\prime 2}-2 f(r)^{\prime \prime} f(r)}{4 r^{2}}-1-\frac{r^{2} p(r) p(r)^{\prime \prime}}{2}-\frac{3 f(r) f(r)^{\prime}}{2 r^{3}}+\frac{f(r)^{2}}{r^{4}}\right.\right. \\
& \left.\left.-\frac{r^{2} p(r)^{\prime 2}}{4}-\frac{3 r p(r) p(r)^{\prime}}{2}\right] \sin ^{2} \theta+4\left(2-2 f(r)+f(r)^{\prime} r\right)\right\} a^{2},
\end{aligned}
$$




$$
\begin{aligned}
R^{i j}{ }_{i j} & =\bar{R}^{i j}{ }_{i j}+\tilde{R}^{i j}{ }_{i j}, \quad \bar{R}^{i j}{ }_{i j}=\frac{1-f(r)}{r^{2}}, \quad(3 \leq i<j \leq D), \\
\tilde{R}_{34}^{34} & =\left\{\left[\frac{6-5 f(r)-f(r)^{2}}{r^{4}}+\frac{f(r) f(r)^{\prime}}{2 r^{3}}+\frac{10-r p(r) p(r)^{\prime}}{2}\right] \sin ^{2} \theta\right. \\
& \left.-\frac{9}{r^{2} l^{2}}-\frac{6(1-f(r))}{r^{4}}\right\} a^{2}, \\
\tilde{R}_{3 k}^{3 k} & =2 a^{2} \cos ^{2} \theta\left(-\frac{1}{r^{2} l^{2}}+\frac{f(r)-1}{r^{4}}\right), \quad(5 \leq k \leq D) \\
\tilde{R}_{4 l}^{4 l} & =\left\{\left[\frac{f(r) f(r)^{\prime}}{2 r^{3}}-\frac{r p(r) p(r)^{\prime}}{2}+\frac{3}{r^{2} l^{2}}+\frac{-f(r)^{2}+2-f(r)}{r^{4}}\right] \sin ^{2} \theta\right. \\
& \left.+\frac{2(f(r)-1)}{r^{4}}-\frac{2}{r^{2} l^{2}}\right\} a^{2}, \quad(5 \leq l \leq D), \\
\tilde{R}_{k l}^{k l} & =a^{2} \cos ^{2} \theta \frac{f(r)-1}{r^{4}}, \quad(5 \leq k<l \leq D) .
\end{aligned}
$$

group (II):

$$
\begin{aligned}
& R_{34}^{12}=-a r^{2} \sin \theta \cos \theta p^{\prime}(r), \quad R_{24}^{13}=-\frac{a \cos \theta \sin \theta p^{\prime}(r)}{2 f(r)}, \\
& R_{32}^{14}=-\frac{a \cos \theta p^{\prime}(r)}{2 f(r) \sin \theta}, \quad R_{24}^{12}=-\frac{a r \sin ^{2} \theta}{2}\left(3 p^{\prime}(r)+r p^{\prime \prime}(r)\right), \\
& R_{i 4}^{1 i}=-\frac{a r \sin ^{2} \theta}{2} p^{\prime}(r), \quad(3 \leq i \leq D) .
\end{aligned}
$$

group (III):

$$
\begin{aligned}
R_{13}^{12} & =\left[-\frac{f^{\prime}(r)}{2 f(r) r^{2}}-p(r)^{2} r-\frac{3}{r^{3}}+\frac{p(r)^{2} f^{\prime}(r) r^{2}}{2 f(r)}-p(r) p^{\prime}(r) r^{2}\right. \\
& \left.+\frac{3 f(r)}{r^{3}}-\frac{f^{\prime}(r)}{r^{2}}\right] a^{2} \sin \theta \cos \theta \\
R^{24}{ }_{34} & =\left[-p(r) p^{\prime}(r) r^{2}+\frac{3 f(r)(1-f(r))}{r^{3}}+\frac{3 f^{\prime}(r) f(r)}{2 r^{2}}\right] a^{2} \sin \theta \cos \theta .
\end{aligned}
$$

With regard to Ricci scalar, the term $\tilde{R}$ which is proportional to $a^{2}$ is given by

$$
\begin{aligned}
R & =\left\{\left[\left(\frac{r^{2} f(r)^{\prime \prime}}{f(r)}-8-\frac{r^{2} f(r)^{\prime 2}}{2 r^{2} f(r)^{2}}+\frac{4 r f(r)^{\prime}}{f(r)}+\frac{8}{f(r)}\right) p(r)^{2}+\left(14 r-\frac{r^{2} f(r)^{\prime}}{f(r)}\right) p(r)^{\prime}\right.\right. \\
& -\frac{3 r^{2} p(r)^{\prime 2}}{2}-\frac{2 f(r)^{\prime}}{f(r) r^{3}}-\frac{2 f(r) f(r)^{\prime}}{r^{3}}+\frac{f(r)^{\prime 2}}{r^{2}}-\frac{8}{r^{4} f(r)}+\frac{f(r) f(r)^{\prime \prime}}{r^{2}}-\frac{2 f(r)^{\prime}}{r^{3}} \\
& \left.+\frac{f(r)^{\prime 2}}{2 f(r)^{2} r^{2}}-\frac{f(r)^{\prime \prime}}{r^{2}}-\frac{2 f(r)^{2}}{r^{4}}+\frac{20}{r^{4}}-\frac{f(r)^{\prime \prime}}{f(r) r^{2}}-\frac{10 f(r)}{r^{4}}\right] \sin ^{2} \theta \frac{4 p(r)^{2}}{f(r)}-\frac{14}{r^{4}}+\frac{4}{f(r) r^{4}} \\
& \left.+\frac{6 f(r)^{\prime}}{r^{3}}+\frac{f(r)^{\prime \prime}}{r^{2}}+\frac{10 f(r)}{r^{4}}+\frac{30 \sin ^{2} \theta-26}{r^{2} l^{2}}\right\} a^{2} .
\end{aligned}
$$


Acknowledgment This work has been supported by the Natural Science Foundation of China under grant Nos.10875060, 10975180 and 11047025.

[1] Myers R C and Simon J Z 1988 Phys. Rev. D 382434.

[2] Stelle K S 1977 Phys. Rev. D 16953.

[3] Berredo-Peixoto G de and Shapiro I L 2004 Phys. Rev. D 70044024.

[4] Berredo-Peixoto G de and Shapiro I L 2005 Phys. Rev. D 71064005.

[5] Lust D and Theisen S 1989 Lect. Notes Phys. 3461.

[6] Myers R C 1987 Phys. Rev. D 36392.

[7] Alekseev S O and Pomazanov M V 1997 Grav. Cosmol. 3191.

[8] Maeda K i, Ohta N and Sasagawa Y 2009 Phys. Rev. D 80104032.

[9] Lovelock D 1971 J. Math. Phys. 12498.

[10] Zwiebach B 1985 Phys. Lett. B 156315.

[11] Zumino B 1986 Phys. Rept. 137109.

[12] Boulware D G and Deser S 1985 Phys. Rev. Lett. 552656.

[13] Wheeler J T 1986 Nucl. Phys. B 273732.

[14] Cai R G and Guo Q 2004 Phys. Rev. D 69104025.

[15] Cai R G 2002 Phys. Rev. D 65084014

[16] Cho Y M and Neupane I P 2002 Phys. Rev. D 66024044.

[17] Cvetic M, Nojiri S and Odintsov S D 2002 Nucl. Phys. B 628295.

[18] Chen J and Wang Y 2010 Chin. Phys. B 19060401.

[19] Liu J 2007 Commun. Theor. Phys. 47647.

[20] Dehghani M H and Pourhasan R 2009 Phys. Rev. D 79064015.

[21] Ge X H, Sin S J, Wu S F and Yang G H 2009 Phys. Rev. D 80104019.

[22] Zou D C, Yue R H and Yang Z Y 2011 Commun. Theor. Phys. 55449.

[23] Zou D C, Yang Z Y and Yue R H 2011 Chin. Phys. Lett. 28020402.

[24] Aliev A N 2007 Phys. Rev. D 75084041.

[25] Aliev A N 2006 Phys. Rev. D 74024011.

[26] Komar A 1959 Phys. Rev. 113934.

[27] Peng J J and Wu S Q 2008 Chin. Phys. B 17825.

[28] Zeng X X, Yang S Z and Chen D Y 2008 Chin. Phys. B 171629. 